TTP12-012

$\mathrm{SFB} / \mathrm{CPP}-12-23$

\title{
Three-loop $\beta$-functions for top-Yukawa and the Higgs self-interaction in the Standard Model
}

\author{
K. G. Chetyrkin ${ }^{a}$, M. F. Zoller ${ }^{a}$ \\ ${ }^{a}$ Institut für Theoretische Teilchenphysik, Karlsruhe Institute of Technology (KIT), D-76128 \\ Karlsruhe, Germany
}

\begin{abstract}
We analytically compute the dominant contributions to the $\beta$-functions for the top-Yukawa coupling, the strong coupling and the Higgs self-coupling as well as the anomalous dimensions of the scalar, gluon and quark fields in the unbroken phase of the Standard Model at threeloop level. These are mainly the QCD and top-Yukawa corrections. The contributions from the Higgs self-interaction which are negligible for the running of the top-Yukawa and the strong coupling but important for the running of the Higgs self-coupling are also evaluated.
\end{abstract}

\section{Introduction}

Using perturbation theory in any renormalizable quantum field theory comes with the price that the parameters of this theory, e.g. the couplings and masses, in general depend on the renormalization scale $\mu$. The precise description of the evolution of these parameters with the energy scale is an important task in any model. This is done by means of the Renormalization Group functions, that is the $\beta$-functions and anomalous dimensions. The knowledge of the three-loop contributions to the $\beta$-functions for the Standard Model (SM) and its extensions is important for physics at the very high energy frontier and for cosmology. Here are some examples: The running of the gauge couplings plays an important role for the construction of Grand Unified theories of the strong and electroweak interactions. The $\beta$-functions for the scalar self-interaction and for the top quark Yukawa coupling constant are important for the analysis of Higgs-inflation in the SM 1 14. The current investigations of these issues are based on the two-loop approximation. The inclusion of the next order could be essential. Further, in a recent work [5], the possibility has been discussed that the SM, supplemented by the asymptotically safe gravity could play the role of a fundamental, rather than effective field theory. Within this framework the mass of the Higgs boson has been predicted to be approximately $126 \mathrm{GeV}$. The theoretical uncertainty of the prediction is about $2 \mathrm{GeV}$.

Recent exciting evidence from several SM-like Higgs search channels at both the CERN Large Hadron Collider and the Fermilab Tevatron [6] point to the possibility of a SM Higgs boson with a mass in the vicinity of $125 \mathrm{GeV}$ which is in truly remarkable agreement with the aforementioned prediction. 1 This calls for more precise calculations, in particular of $\beta$-functions, in the SM. In the present paper we are particularly interested in the evolution of the Higgs self-coupling as well as the top-Yukawa coupling in the SM.

\footnotetext{
${ }^{1}$ Note that the boundary condition $\lambda\left(M_{\text {Planck }}\right)=0$, leading to the prediction of the Higgs mass close the the experimental evidence, has been also discussed recently in [9].
} 
The underlying gauge group of the $\mathrm{SM}$ is an $\mathrm{SU}_{C}(3) \times \mathrm{SU}(2) \times \mathrm{U}_{Y}(1)$ which is spontaneously broken to $\mathrm{SU}_{C}(3) \times \mathrm{U}_{Q}(1)$ at the electroweak scale. As the renormalization constants for fields and vertices do not depend on masses and external momenta in the $\overline{\mathrm{MS}}$-scheme, we will perform our calculations in the unbroken phase of the SM.

The most important contributions to the running of the Higgs self-coupling $\lambda$ arise from the top-Yukawa coupling and the strong sector. All other Yukawa couplings are significantly smaller due to to the smallness of the respective quark masses. From the top mass $M_{t} \approx 172.9 \mathrm{GeV}$ we get the Yukawa coupling at this scale $y_{t}\left(M_{t}\right)=\sqrt{2} \frac{M_{t}}{v} \approx 1$ where $v \approx 246.2 \mathrm{GeV}$ is proportional to the vacuum expectation value of the scalar field $\Phi$ from which results the Higgs field after the spontaneous symmetry breaking: $|\langle\Phi\rangle|=\frac{v}{\sqrt{2}}$.

The next Yukawa coupling to be considered would be $y_{b}=\sqrt{2} \frac{M_{b}}{v} \approx 0.02$. The strong coupling at the scale of the $\mathrm{Z}$ boson mass is $g_{s}\left(M_{Z}\right) \approx 1.22$ whereas the electroweak couplings

$g_{1}\left(M_{Z}\right)=\frac{\sqrt{4 \pi \alpha}}{\cos \theta_{W}} \approx 0.36$ and $g_{2}\left(M_{Z}\right)=\frac{\sqrt{4 \pi \alpha}}{\sin \theta_{W}} \approx 0.65$ give much smaller contributions which are further suppressed by the isospin and hypercharge factors. For this reason we will consider a simplified version of the SM or - as one could also see it - a minimal extension of QCD by setting $g_{1}=g_{2}=0$ in our calculation. For a Higgs mass of $125 \mathrm{GeV}$ the value of the Higgs self-interaction would be $\lambda\left(M_{H}\right) \approx 0.13$ at the scale of the Higgs mass. The relevance of this parameter will be examined in section 2 .

The outline of the work is as follows. In the next section we discuss the main definitions and the general setup of our work. Section 3 deals with the technical details, including the treatment of $\gamma_{5}$. In sections 4 and 5 we present our results for the $\beta$-functions of the topYukawa, the strong and the Higgs self-couplings and the relevant field anomalous dimensions. The numerical influence of the computed three-loop corrections on the evolution of the quartic Higgs coupling is discussed in section 6. For this analysis we will include the already known contributions with $g_{1}$ and $g_{2}$ at one-loop and two-loop level. Finally, section 7 contains our conclusions and acknowledgements.

All our results for $\beta$-functions and anomalous dimensions can be retrieved from

http://www-ttp.particle.uni-karlsruhe.de/Progdata/ttp12/ttp12-012/

\section{General Setup}

The Lagrangian of our model consists of three pieces:

$$
\mathcal{L}=\mathcal{L}_{Q C D}+\mathcal{L}_{y_{t}}+\mathcal{L}_{\Phi}
$$

The QCD part is defined by

$$
\begin{aligned}
\mathcal{L}_{Q C D}= & -\frac{1}{4} G_{\mu \nu}^{a} G^{a \mu \nu}-\frac{1}{2(1-\xi)}\left(\partial_{\mu} A^{a \mu}\right)^{2}+\partial_{\mu} \bar{c}^{a} \partial^{\mu} c^{a}+g_{s} f^{a b c} \partial_{\mu} \bar{c}^{a} A^{b \mu} c^{c} \\
& +\sum_{q}\left\{\frac{i}{2} \bar{q} \overleftrightarrow{\phi} q+g_{s} \bar{q} A^{a} T^{a} q\right\}
\end{aligned},
$$

where $q$ runs over all quark flavours, the gluon field strength tensor is given by

$$
G_{\mu \nu}^{a}=\partial_{\mu} A_{\nu}^{a}-\partial_{\nu} A_{\mu}^{a}+g_{s} f^{a b c} A_{\mu}^{b} A_{\nu}^{c}
$$

and $f^{a b c}$ are the structure constants of the colour gauge group with the generators $T^{a}$ :

$$
\left[T^{a}, T^{b}\right]=i f^{a b c} T^{c}
$$


The complex scalar field $\Phi$ and the left-handed parts of the top and bottom quarks $t_{L}$ and $b_{L}$ are doublets under $\mathrm{SU}(2)$ :

$$
\Phi=\left(\begin{array}{c}
\Phi_{1} \\
\Phi_{2}
\end{array}\right), \quad Q_{L}=\left(\begin{array}{c}
t \\
b
\end{array}\right)_{L} .
$$

Setting all Yukawa couplings to zero except for the top coupling $y_{t}$ the Lagrangian for the Yukawa sector is given by

$$
\begin{aligned}
\mathcal{L}_{y_{t}} & =-y_{t}\left\{\bar{t}_{R} \Phi^{\dagger c} Q_{L}+\bar{Q}_{L} \Phi^{c} t_{R}\right\} \\
& =-y_{t}\left\{\bar{t}_{R}\left(\Phi_{2},-\Phi_{1}\right) \cdot\left(\begin{array}{c}
t \\
b
\end{array}\right)_{L}+(\bar{t}, \bar{b})_{L} \cdot\left(\begin{array}{c}
\Phi_{2}^{*} \\
-\Phi_{1}^{*}
\end{array}\right) t_{R}\right\} \\
& =-y_{t}\left\{\left(\bar{t} P_{R} t\right) \Phi_{2}^{*}+\left(\bar{t} P_{L} t\right) \Phi_{2}-\left(\bar{b} P_{R} t\right) \Phi_{1}^{*}-\left(\bar{t} P_{L} b\right) \Phi_{1}\right\} .
\end{aligned}
$$

Finally, we have the scalar sector of the model

$$
\mathcal{L}_{\Phi}=\partial_{\mu} \Phi^{\dagger} \partial^{\mu} \Phi-m^{2} \Phi^{\dagger} \Phi-\lambda\left(\Phi^{\dagger} \Phi\right)^{2} .
$$

The indices $\mathrm{L}$ and $\mathrm{R}$ indicate the left- and right-handed part of the fields as obtained by the projectors

$$
P_{L}=\frac{1}{2}\left(1-\gamma_{5}\right) \quad P_{R}=\frac{1}{2}\left(1+\gamma_{5}\right) .
$$

This model is renormalized with the counterterm Lagrangians

$$
\begin{aligned}
\delta \mathcal{L}_{Q C D}= & -\frac{1}{4} \delta Z_{3}^{(2 g)}\left(\partial_{\mu} A_{\nu}^{a}-\partial_{\nu} A_{\mu}^{a}\right)^{2}-\frac{1}{2} \delta Z_{1}^{(3 g)} g_{s} f^{a b c}\left(\partial_{\mu} A_{\nu}^{a}-\partial_{\nu} A_{\mu}^{a}\right) A_{\mu}^{b} A_{\nu}^{c} \\
& -\frac{1}{4} \delta Z_{1}^{(4 g)} g_{s}^{2}\left(f^{a b c} A_{\mu}^{b} A_{\nu}^{c}\right)^{2}+\delta Z_{3}^{(2 c)} \partial_{\mu} \bar{c}^{a} \partial^{\mu} c^{a}+\delta Z_{1}^{(c c g)} g_{s} f^{a b c} \partial_{\mu} \bar{c}^{a} A^{b \mu} c^{c} \\
& +\sum_{q}\left\{\frac{i}{2} \bar{q} \overleftrightarrow{\not}\left[\delta Z_{2, L}^{(2 q)} P_{L}+\delta Z_{2, R}^{(2 q)} P_{R}\right] q+g_{s} \bar{q} A^{a} T^{a}\left[\delta Z_{1, L}^{(q q g)} P_{L}+\delta Z_{1, R}^{(q q g)} P_{R}\right] q\right\}
\end{aligned}
$$

for the QCD part,

$$
\delta \mathcal{L}_{\text {Yukawa }}=-\delta Z_{1}^{(t b \Phi)} y_{t}\left\{\left(\bar{t} P_{R} t\right) \Phi_{2}^{*}+\left(\bar{t} P_{L} t\right) \Phi_{2}-\left(\bar{b} P_{R} t\right) \Phi_{1}^{*}-\left(\bar{t} P_{L} b\right) \Phi_{1}\right\}
$$

for the Yukawa part and

$$
\delta \mathcal{L}_{\Phi}=\delta Z_{2}^{(2 \Phi)} \partial_{\mu} \Phi^{\dagger} \partial^{\mu} \Phi-m^{2} \delta Z_{\Phi^{2}} \Phi^{\dagger} \Phi+\delta Z_{1}^{(4 \Phi)}\left(\Phi^{\dagger} \Phi\right)^{2}
$$

for the $\Phi$-sector. Note that in general the left- and right-handed parts of quark fields and quark-vertices are renormalized differently 2 So the renormalization constant for the strong gauge coupling $g_{s}$ can be obtained for example from

$$
Z_{g_{s}}=\frac{Z_{1, L}^{(t t g)}}{Z_{2, L}^{(2 t)} \sqrt{Z_{3}^{(2 g)}}}=\frac{Z_{1, R}^{(t t g)}}{Z_{2, R}^{(2 t)} \sqrt{Z_{3}^{(2 g)}}}
$$

or the renormalization constant for $y_{t}$ from

$$
Z_{y_{t}}=\frac{Z_{1}^{(t b \Phi)}}{\sqrt{Z_{2, L}^{(2 t)} Z_{2, R}^{(2 t)} Z_{2}^{(2 \Phi)}}} .
$$

\footnotetext{
${ }^{2}$ In our case this is true for the quark fields participating in the Yukawa sector. As we do not consider the electroweak interaction here and neglect all Yukawa couplings except for $y_{t}$ the light quark fields $\mathrm{u}, \mathrm{d}, \mathrm{s}$ and $\mathrm{c}$ have the same renormalization constant for the left- and right-handed part.
} 
Here the renormalization constants have been defined in a minimal way as

$$
Z=1+\delta Z
$$

with $\delta Z$ containing only poles in the regulating parameter $\varepsilon=(4-D) / 2$ of the dimensional regularization and $D$ being the engineering space-time dimension. The Higgs self-coupling $\lambda$ is related to the Higgs mass at tree level $M_{H}$ via

$$
\lambda=\frac{M_{H}^{2}}{2 v^{2}},
$$

which for $M_{H}=125 \mathrm{GeV}$ yields $\lambda\left(M_{H}=125 \mathrm{GeV}\right) \approx 0.13$. For the running of the topYukawa coupling the contribution from $\lambda$ is negligible compared to the top-Yukawa and strong coupling. The corresponding four- $\Phi$ vertex is nevertheless needed for the renormalization at three-loop level, namely to kill the subdivergence from the fermion loop in diagrams like Fig. 1(d). The $\beta$-function for a coupling $\mathrm{X}$ is defined as

$$
\beta_{X}=\mu^{2} \frac{d X}{d \mu^{2}}=\sum_{n=1}^{\infty} \frac{1}{\left(16 \pi^{2}\right)^{n}} \beta_{X}^{(n)}
$$

and is given as a power series in all couplings of the model, namely $g_{s}, y_{t}$ and $\lambda$. Note that the $\beta$-functions $\beta_{g_{s}}$ and $\beta_{y_{t}}$ are proportional to $g_{s}$ and $y_{t}$ respectively whereas $\beta_{\lambda}$ has one part proportional to $\lambda$ and one part proportional to $y_{t}^{4}$ with no $\lambda$-dependence at all. The anomalous dimension of a field $f$ is defined as

$$
\gamma_{2}^{f}=-\mu^{2} \frac{d \ln Z_{f}^{-1}}{d \mu^{2}}=\sum_{n=1}^{\infty} \frac{1}{\left(16 \pi^{2}\right)^{n}} \gamma_{2}^{f(n)}
$$

where $Z_{f}$ is the field strength renormalization constant for the respective field 3 The $\beta$ functions for all couplings are independent of the gauge parameter $\xi$ whereas the anomalous dimensions of the fields are not.

\section{Calculation}

As we are interested in $\beta$-functions it is enough to compute the UV divergent part of all diagrams in order to determine the necessary renormalization constants. In the $\overline{\mathrm{MS}}$-scheme the latter depends only polynomially on external momenta and masses. Therefore most of our renormalization constants could be computed from massless propagator-like diagrams using the FORM 3 [10] version 4 of the package MINCER [11]. In some diagrams, e.g. with four external $\Phi$-fields where two external momenta are set to zero, this leads to IR divergences which mix with the UV ones in dimensional regularization. Another convenient method to compute renormalization constants has been suggested in [12] and elaborated in the context of three-loop calculations in [13. The idea is an exact decomposition of all propagators using an auxiliary mass parameter $M^{2}$ :

$$
\frac{1}{(q+p)^{2}}=\frac{1}{q^{2}-M^{2}}+\frac{-p^{2}-2 q \cdot p-M^{2}}{q^{2}-M^{2}} \frac{1}{(q+p)^{2}}
$$

\footnotetext{
${ }^{3}$ For an $n$-point vertex $V$ or a mass $m$ the anomalous dimension is defined as $\gamma_{n}^{V}=-\mu^{2} \frac{d \ln Z_{V}}{d \mu^{2}}$ or $\gamma_{m}=-\mu^{2} \frac{d \ln Z_{m}}{d \mu^{2}}$. For a field we take the inverse renormalization constant.

${ }^{4}$ The program can be downloaded from
}

http://www.nikhef.nl/〜 form/maindir/packages/mincer/mincer.html 
where $p$ is a combination of external and $q$ of internal momenta. This can be done recursively until the power in the denominator of the last term is high enough for this contribution to be finite, e.g.

$$
\begin{aligned}
\frac{1}{(q+p)^{2}}= & \frac{1}{q^{2}-M^{2}}+\frac{-p^{2}-2 q \cdot p}{\left(q^{2}-M^{2}\right)^{2}}+\frac{\left(-p^{2}-2 q \cdot p\right)^{2}}{\left(q^{2}-M^{2}\right)^{3}}-\frac{M^{2}}{\left(q^{2}-M^{2}\right)^{2}} \\
& +\frac{M^{2}\left(M^{2}+2 p^{2}+4 q \cdot p\right)}{\left(q^{2}-M^{2}\right)^{3}}+\frac{\left(-p^{2}-2 q \cdot p-M^{2}\right)^{3}}{\left(q^{2}-M^{2}\right)^{3}} \frac{1}{(q+p)^{2}} .
\end{aligned}
$$

As the result is independent of $M^{2}$ we can omit the contributions $\sim M^{2}$ in the above decomposition as long as we introduce counterterms into the Lagrangian to cancel $M^{2}$-dependent subdivergences. In our case only a term $\frac{M^{2}}{2} \delta Z_{M^{2}}^{(2 g)} A_{\mu}^{a} A^{a \mu}$ and $\frac{M^{2}}{2} \delta Z_{M^{2}}^{(2 \Phi)} \Phi^{\dagger} \Phi$ are possible 5 The first one is not gauge invariant but this does not matter as it is only used for the cancellation of subdivergences which works nevertheless. This method effectively amounts to introducing the same auxiliary mass parameter $M^{2}$ in every denominator of propagators and all possible $M^{2}$-counterterms. We expand in the external momenta6 and arrive at massive tadpole diagrams with one scale $M$. Due to the auxiliary mass no IR divergences can appear while the UV counterterms which we are interested in (that is the ones without any dependence on the auxiliary mass M) will stay untouched.

For the calculation of massive tadpoles we have used the FORM-based program MATAD [14]. Where possible, i.e. for the propagators and three point functions, we have employed both the MINCER and the MATAD setups which served as an extra check. To generate the diagrams we used QGRAF [15] and to compute the colour factors the FORM package COLOR [16].

An important aspect of calculations such as these is the proper treatment of $\gamma_{5}$. As is well known, a naive treatment of $\gamma_{5}$ can be applied if it only appears in an external fermion line. In fermion loops we have to be more careful. In four dimensions we define

$$
\gamma_{5}=i \gamma^{0} \gamma^{1} \gamma^{2} \gamma^{3}=\frac{i}{4 !} \varepsilon_{\mu \nu \rho \sigma} \gamma^{\mu} \gamma^{\nu} \gamma^{\rho} \gamma^{\sigma} \text { with } \varepsilon_{0123}=1=-\varepsilon^{0123}
$$

In Fig. 1 to 5 we show a few diagrams that had to be calculated for the various ingredients of our final result. In order to have a contribution from a fermion loop with one $\gamma_{5}$ in it at least four free Lorentz indices or momenta on the external lines of the minimal subgraph containing this fermion loop are required. These can be indices from the gluon vertices or the internal momenta from other loops which act as external momenta to the minimal subgraph containing the fermion loop in question. External momenta of the whole diagram can be set to zero as the renormalization constants in the $\overline{\mathrm{MS}}$-scheme do not depend on those.

Consider for example one of the fermion loops in Fig[2 (c). The momenta on the two external $\Phi$-legs can be set to zero. Then we have two indices from the gluon lines attached to our fermion loop and one loop momentum going through the two gluons and acting as an external momentum to the subgraph containing only our fermion loop. This is not enough to have a non-naive $\gamma_{5}$ contribution from this graph.

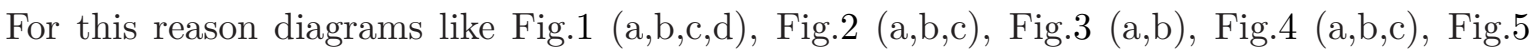
$(\mathrm{a}, \mathrm{b})$ can be treated naively. Fig 3 (c) has enough indices and momenta but no $\gamma_{5}$ in it.

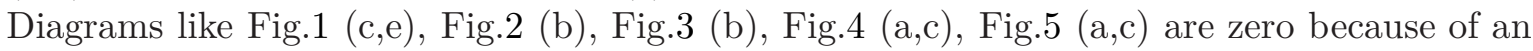
odd number of $\gamma$-matrices in at least one fermion loop. And diagrams like Fig[1(b), Fig_2 (b),

\footnotetext{
${ }^{5}$ Counterterms $\sim M$ that would arise for fermions cannot appear because we have no $M$ in the numerators of propagators. The ghost mass term $\frac{M^{2}}{2} \delta Z_{M^{2}}^{(2 c)} \bar{c}^{a} c^{a}$ does not appear because of the momentum dependence of the ghost-gluon-vertex.

${ }^{6}$ This method also works in massive theories. In this case we expand in the physical masses as well.
} 
Fig 3 (a), Fig 4 (b), Fig 5 (b) are zero because of their colour structure. The only problematic type is Fig 1(f) which fortunately only contributes a $\frac{1}{\varepsilon}$ pole and can therefore be treated as described in [17. We use the fact that $\gamma_{5}$ anticommutes with every other $\gamma$-matrix in four dimensions and that $\gamma_{5}^{2}=1$. Then we apply relation (20) for the case when one $\gamma_{5}$ remains on each fermion line. The two $\varepsilon_{\mu \nu \rho \sigma}$ can be rewritten as a combination of metric tensors which can be handled in dimensional regularization. The error we make with this treatment is of order $\varepsilon$ and does therefore not affect the pole part of our result.
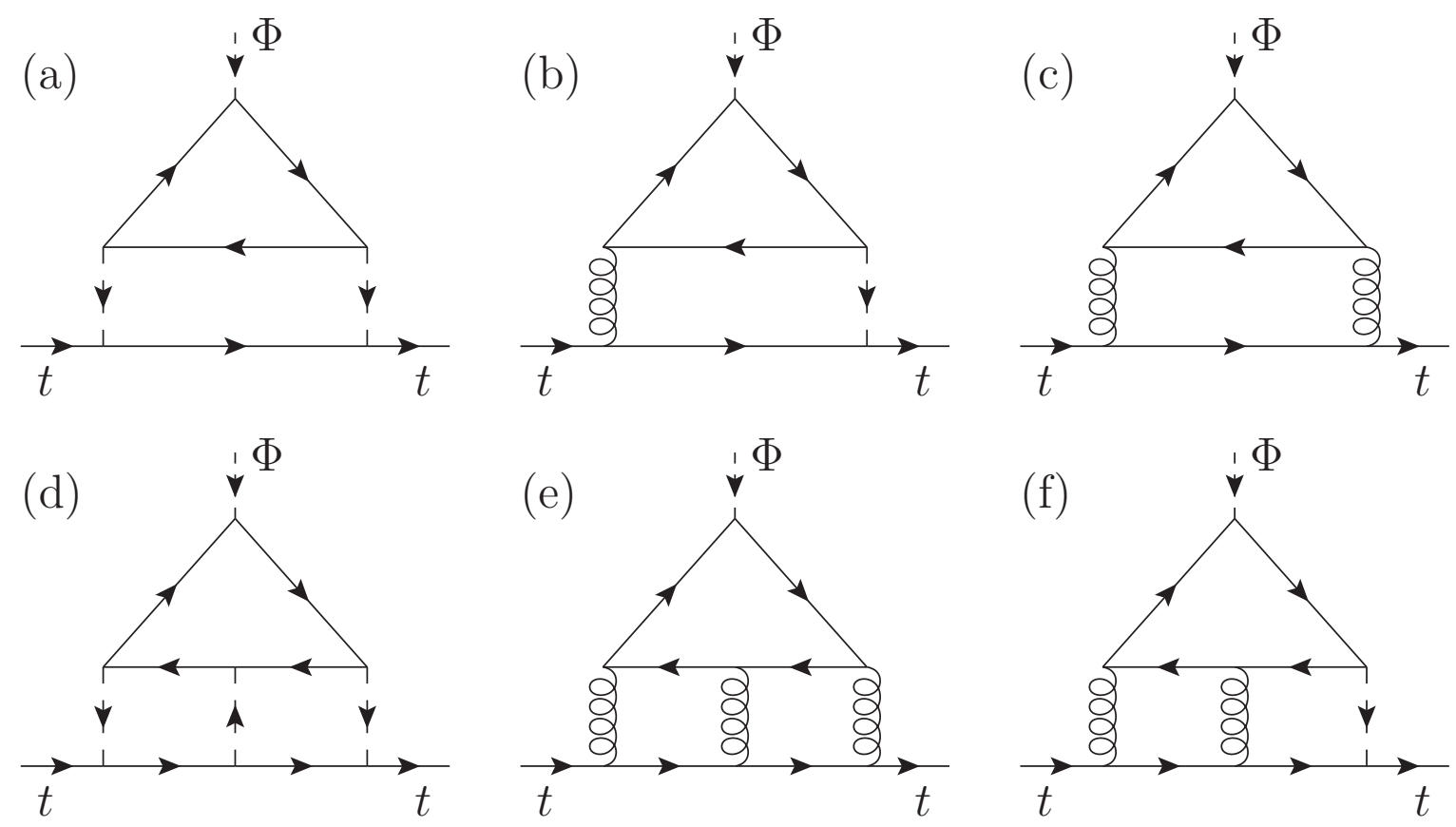

Figure 1: Some diagrams contributing to $Z_{1}^{(t b \Phi)}$

(a)

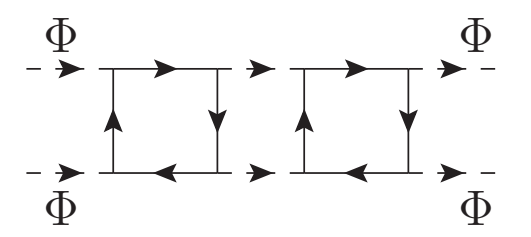

(b)

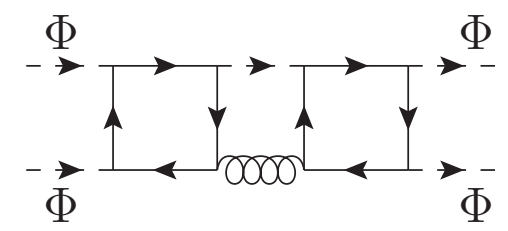

(c)

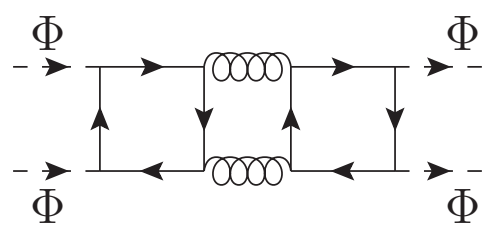

Figure 2: Some diagrams contributing to $Z_{1}^{(4 \Phi)}$

\section{Results for the $\beta$-functions}

First we give the results for the three-loop $\beta$-functions of couplings $\lambda, y_{t}$ and $g_{s}$ with the general gauge group factors for the strong interacting sector. Below $C_{F}$ and $C_{A}$ are the quadratic Casimir operators of the quark and the adjoint representation of the corresponding Lie algebra, $d_{R}$ is the dimension of the quark representation, $T_{F}$ is defined so that $T_{F} \delta^{a b}=\operatorname{Tr}\left(T^{a} T^{b}\right)$ is the trace of two group generators of the quark representation 7 For QCD (colour gauge

\footnotetext{
${ }^{7}$ For an $\mathrm{SU}(N)$ gauge group these are $d_{R}=N, C_{A}=2 T_{F} N$ and $C_{F}=T_{F}\left(N-\frac{1}{N}\right)$.
} 
(a)

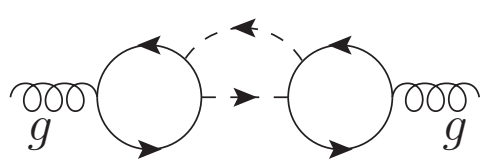

(b)

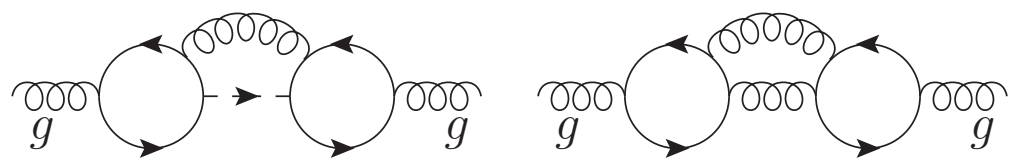

(c)

Figure 3: Some diagrams contributing to $Z_{3}^{(2 g)}$

(a)

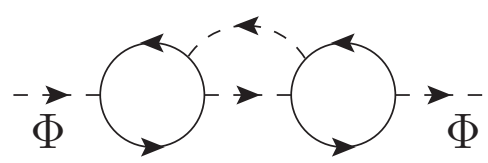

(b)

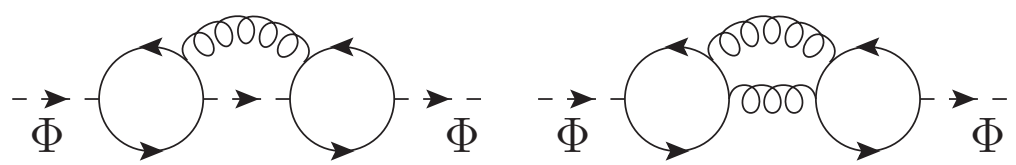

(c) $\Phi$

Figure 4: Some diagrams contributing to $Z_{2}^{(2 \Phi)}$

(a)

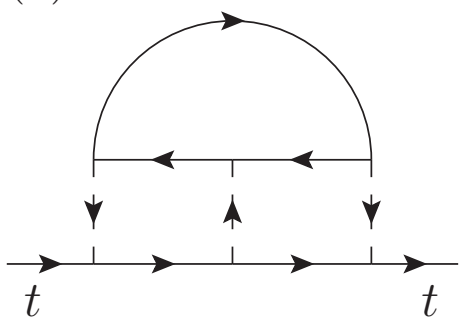

(b)

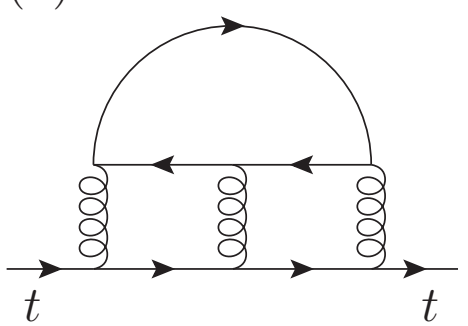

Figure 5: Some diagrams contributing to $Z_{2, L / R}^{(2 t)}$

group SU(3)) we have $C_{F}=4 / 3, C_{A}=3, T_{F}=1 / 2$ and $d_{R}=3$. Furthermore we denote the 
number of fermions by $n_{f}=n_{l}+1$.

$$
\begin{aligned}
\beta_{\lambda}^{(1)}= & 12 \lambda^{2}+2 d_{R} y_{t}^{2} \lambda-d_{R} y_{t}^{4}, \\
\beta_{\lambda}^{(2)}= & -156 \lambda^{3}-24 d_{R} y_{t}^{2} \lambda^{2}-\frac{1}{2} d_{R} y_{t}^{4} \lambda+5 d_{R} y_{t}^{6} \\
& +10 C_{F} d_{R} g_{s}^{2} y_{t}^{2} \lambda-4 C_{F} d_{R} g_{s}^{2} y_{t}^{4}, \\
\beta_{\lambda}^{(3)}= & \lambda^{4}\left(3588+2016 \zeta_{3}\right)+291 d_{R} y_{t}^{2} \lambda^{3}+y_{t}^{4} \lambda^{2}\left(\frac{789}{2} d_{R}+252 \zeta_{3} d_{R}-36 d_{R}^{2}\right) \\
& +y_{t}^{6} \lambda\left(-\frac{1881}{8} d_{R}-66 \zeta_{3} d_{R}+80 d_{R}^{2}\right)+y_{t}^{8}\left(\frac{13}{2} d_{R}-12 \zeta_{3} d_{R}-\frac{195}{8} d_{R}^{2}\right) \\
& +g_{s}^{2} y_{t}^{2} \lambda^{2}\left(-306 C_{F} d_{R}+288 \zeta_{3} C_{F} d_{R}\right)+g_{s}^{2} y_{t}^{4} \lambda\left(\frac{895}{4} C_{F} d_{R}-324 \zeta_{3} C_{F} d_{R}\right) \\
& +g_{s}^{2} y_{t}^{6}\left(-\frac{19}{2} C_{F} d_{R}+60 \zeta_{3} C_{F} d_{R}\right)+g_{s}^{4} y_{t}^{2} \lambda\left(-\frac{119}{2} C_{F}^{2} d_{R}+77 C_{A} C_{F} d_{R}\right. \\
& \left.-16 n_{f} T_{F} C_{F} d_{R}+72 \zeta_{3} C_{F}^{2} d_{R}-36 \zeta_{3} C_{A} C_{F} d_{R}\right)+g_{s}^{4} y_{t}^{4}\left(\frac{131}{2} C_{F}^{2} d_{R}\right. \\
& \left.+48 T_{F} C_{F} d_{R}-\frac{109}{2} C_{A} C_{F} d_{R}+10 n_{f} T_{F} C_{F} d_{R}-48 \zeta_{3} C_{F}^{2} d_{R}+24 \zeta_{3} C_{A} C_{F} d_{R}\right)
\end{aligned}
$$

The purely $\lambda$-dependent parts of eq. (21) have been known for a while [18, 19], the full oneloop and two-loop result are in agreement with [20,21] (for an $\mathrm{SU}(3)$ colour gauge group). It has also been a useful check for our setup to see that the same result can be derived from the four- $\Phi_{1}$ vertex, the four- $\Phi_{2}$ vertex and the $\left(\Phi_{1}^{*} \Phi_{2}^{*} \Phi_{1} \Phi_{2}\right)$ vertex.

$$
\begin{aligned}
\frac{\beta_{y_{t}}^{(1)}}{y_{t}}= & y_{t}^{2}\left(\frac{3}{4}+\frac{1}{2} d_{R}\right)-3 C_{F} g_{s}^{2}, \\
\frac{\beta_{y_{t}}^{(2)}=}{y_{t}}= & \lambda^{2}-6 y_{t}^{2} \lambda+y_{t}^{4}\left(\frac{3}{4}-\frac{9}{4} d_{R}\right) \\
& +g_{s}^{2} y_{t}^{2}\left(6 C_{F}+\frac{5}{2} C_{F} d_{R}\right)+g_{s}^{4}\left(-\frac{3}{2} C_{F}^{2}-\frac{97}{6} C_{A} C_{F}+\frac{10}{3} n_{f} T_{F} C_{F}\right) \\
\frac{\beta_{y_{t}}^{(3)}=}{y_{t}}= & 18 \lambda^{3}+y_{t}^{2} \lambda^{2}\left(\frac{285}{8}-\frac{45}{4} d_{R}\right)+y_{t}^{4} \lambda\left(\frac{63}{2}+\frac{45}{2} d_{R}\right) \\
& +y_{t}^{6}\left(-\frac{345}{32}+\frac{9}{4} \zeta_{3}+\frac{107}{32} d_{R}+\frac{3}{2} \zeta_{3} d_{R}+\frac{39}{16} d_{R}^{2}\right) \\
& +6 C_{F} g_{s}^{2} y_{t}^{2} \lambda-g_{s}^{2} y_{t}^{4}\left(\frac{57}{2} C_{F}+\frac{81}{8} C_{F} d_{R}\right) \\
& +g_{s}^{4} y_{t}^{2}\left(\frac{471}{16} C_{F}^{2}-\frac{119}{8} C_{F}^{2} d_{R}+25 T_{F} C_{F}+\frac{717}{16} C_{A} C_{F}+\frac{77}{4} C_{A} C_{F} d_{R}\right. \\
& -\frac{33}{4} n_{f} T_{F} C_{F}-4 n_{f} T_{F} C_{F} d_{R}-27 \zeta_{3} C_{F}^{2}+18 \zeta_{3} C_{F}^{2} d_{R}-\frac{27}{2} \zeta_{3} C_{A} C_{F} \\
& \left.-9 \zeta_{3} C_{A} C_{F} d_{R}\right)+g_{s}^{6}\left(-\frac{129}{2} C_{F}^{3}+\frac{129}{4} C_{A} C_{F}^{2}-\frac{11413}{108} C_{A}^{2} C_{F}+46 n_{f} T_{F} C_{F}^{2}\right. \\
& \left.+\frac{556}{27} n_{f} C_{A} T_{F} C_{F}+\frac{140}{27} n_{f}^{2} T_{F}^{2} C_{F}-48 \zeta_{3} n_{f} T_{F} C_{F}^{2}+48 \zeta_{3} n_{f} C_{A} T_{F} C_{F}\right)
\end{aligned}
$$

The one-loop and two-loop part of this result have been found before in [20, 22] (for $d_{R}=3$, $T_{F}=\frac{1}{2}$ ) and the contributions of order $g_{s}^{2}, g_{s}^{4}, y_{t}^{2} g_{s}^{2}$ and $g_{s}^{4} y_{t}$ to $Z_{y_{t}}$ have been successfully checked against [23]. In this reference the calculation of $Z_{M_{t}}$ has been performed in the broken phase of the SM with a massive top quark. When comparing these two results one 
has to take into account that in the broken SM the top quark mass is to be renormalized as a product $y_{t}\left(\Phi_{2}+\Phi_{2}^{\dagger}\right)$ so the corresponding top quark mass renormalization constant is

$$
Z_{M_{t}}=Z_{y_{t}} Z_{2}^{(2 \Phi)}=\frac{Z_{1}^{(t b \Phi)}}{\sqrt{Z_{2, L}^{(2 t)} Z_{2, R}^{(2 t)}}} .
$$

Again the setup could be checked for consistency by using the renormalization of the four different vertices t-t- $\Phi_{2}$, t-t- $\Phi_{2}^{*}$, t-b- $\Phi_{1}$, t-b- $\Phi_{1}^{*}$ for the calculation.

$$
\begin{aligned}
\frac{\beta_{g_{s}}^{(1)}}{g_{s}}= & g_{s}^{2}\left(-\frac{11}{6} C_{A}+\frac{2}{3} n_{f} T_{F}\right), \\
\frac{\beta_{g_{s}}^{(2)}}{g_{s}}= & -2 T_{F} g_{s}^{2} y_{t}^{2}+g_{s}^{4}\left(-\frac{17}{3} C_{A}^{2}+2 n_{f} T_{F} C_{F}+\frac{10}{3} n_{f} C_{A} T_{F}\right), \\
\frac{\beta_{g_{s}}^{(3)}}{g_{s}}= & +g_{s}^{2} y_{t}^{4}\left(\frac{9}{2} T_{F}+\frac{7}{2} T_{F} d_{R}\right)-g_{s}^{4} y_{t}^{2}\left(3 T_{F} C_{F}+12 C_{A} T_{F}\right) \\
& +g_{s}^{6}\left(-\frac{2857}{108} C_{A}^{3}-n_{f} T_{F} C_{F}^{2}+\frac{205}{18} n_{f} C_{A} T_{F} C_{F}+\frac{1415}{54} n_{f} C_{A}^{2} T_{F}\right. \\
& \left.-\frac{22}{9} n_{f}^{2} T_{F}^{2} C_{F}-\frac{79}{27} n_{f}^{2} C_{A} T_{F}^{2}\right) .
\end{aligned}
$$

In the case of $y_{t}=0$ this is in agreement with the well-known result [24,25]. The one-loop and two-loop parts of eq. (23) are known from [26-31], the term $\propto g_{s}^{4} y_{t}^{2}$ can be found in [23] and the full three-loop result has been computed in 32] (for $d_{R}=3, T_{F}=\frac{1}{2}$ ).

The $\beta$-function describing the running of the "mass" parameter $m^{2}$ in eq. (7) can be computed from the renormalization constant of the local operator $O_{2 \Phi}:=\Phi^{\dagger} \Phi$. An insertion of $O_{2 \Phi}$ into a Green's function, e.g. with two external $\Phi$-fields, is renormalized as $\left[O_{2 \Phi}\right]=Z_{\Phi^{2}} O_{2 \Phi}$ where $\left[O_{2 \Phi}\right]$ is the corresponding finite operator. From $\left[O_{2 \Phi}\right]=Z_{m^{2}} O_{2 \Phi}^{\text {bare }}$ and $O_{2 \Phi}^{\text {bare }}=Z_{2}^{(2 \Phi)} O_{2 \Phi}$ it follows that

$$
Z_{m^{2}}=\left(Z_{2}^{(2 \Phi)}\right)^{-1} Z_{\Phi^{2}}
$$

This yields the following contributions to $\beta_{m^{2}}$ :

$$
\begin{aligned}
\frac{\beta_{m^{2}}^{(1)}}{m^{2}}= & 6 \lambda+d_{R} y_{t}^{2} \\
\frac{\beta_{m^{2}}^{(2)}}{m^{2}}= & -30 \lambda^{2}-12 d_{R} y_{t}^{2} \lambda-\frac{9}{4} d_{R} y_{t}^{4}+5 C_{F} d_{R} g_{s}^{2} y_{t}^{2} \\
\frac{\beta_{m^{2}}^{(3)}}{m^{2}}= & 1026 \lambda^{3}+\frac{99}{2} d_{R} y_{t}^{2} \lambda^{2}+y_{t}^{4} \lambda\left(\frac{333}{4} d_{R}-18 d_{R}^{2}+72 \zeta_{3} d_{R}\right) \\
& +y_{t}^{6}\left(-\frac{617}{16} d_{R}+24 d_{R}^{2}+15 \zeta_{3} d_{R}\right) \\
& +g_{s}^{2} y_{t}^{2} \lambda\left(-153 C_{F} d_{R}+144 \zeta_{3} C_{F} d_{R}\right) \\
& +g_{s}^{2} y_{t}^{4}\left(\frac{447}{8} C_{F} d_{R}-90 \zeta_{3} C_{F} d_{R}\right) \\
& +g_{s}^{4} y_{t}^{2}\left(-\frac{119}{4} C_{F}^{2} d_{R}+\frac{77}{2} C_{A} C_{F} d_{R}-8 n_{f} T_{F} C_{F} d_{R}+36 \zeta_{3} C_{F}^{2} d_{R}-18 \zeta_{3} C_{A} C_{F} d_{R}\right)
\end{aligned}
$$

The one-loop and two-loop parts of this result are in agreement with [20] where they have been computed before. The purely $\lambda$-dependent part can be found in [18,19]. For $d_{R}=3$ 
and $T_{F}=\frac{1}{2}$ (QCD) we get the following results:

$$
\begin{aligned}
\beta_{\lambda}^{(1)}= & 12 \lambda^{2}+6 y_{t}^{2} \lambda-3 y_{t}^{4}, \\
\beta_{\lambda}^{(2)}= & -156 \lambda^{3}-72 y_{t}^{2} \lambda^{2}-\frac{3}{2} y_{t}^{4} \lambda+15 y_{t}^{6}+40 g_{s}^{2} y_{t}^{2} \lambda-16 g_{s}^{2} y_{t}^{4}, \\
\beta_{\lambda}^{(3)}= & \lambda^{4}\left(3588+2016 \zeta_{3}\right)+873 y_{t}^{2} \lambda^{3}+y_{t}^{4} \lambda^{2}\left(\frac{1719}{2}+756 \zeta_{3}\right) \\
& +y_{t}^{6} \lambda\left(\frac{117}{8}-198 \zeta_{3}\right)-y_{t}^{8}\left(\frac{1599}{8}+36 \zeta_{3}\right)+g_{s}^{2} y_{t}^{2} \lambda^{2}\left(-1224+1152 \zeta_{3}\right) \\
& +g_{s}^{2} y_{t}^{4} \lambda\left(895-1296 \zeta_{3}\right)+g_{s}^{2} y_{t}^{6}\left(-38+240 \zeta_{3}\right) \\
& +g_{s}^{4} y_{t}^{2} \lambda\left(\frac{1820}{3}-32 n_{f}-48 \zeta_{3}\right)+g_{s}^{4} y_{t}^{4}\left(-\frac{626}{3}+20 n_{f}+32 \zeta_{3}\right) .
\end{aligned}
$$

To get an idea of the size of these contributions and therefore the significance of our calculation we evaluate $\beta_{\lambda}$ at the scale $\mu=M_{Z}$ (with an assumed Higgs mass of $125 \mathrm{GeV}$ and $n_{f}=6$ ) which yields a value of $\beta_{\lambda} \sim(-0.01)$ at one-loop level. The two and three-loop contributions change this result by $\sim 1 \%$ and $\sim(-0.04) \%$ respectively. To estimate the importance of the individual terms we introduce the labels

$$
G=\frac{g_{s}}{g_{s}\left(\mu=M_{Z}\right)}, \quad Y=\frac{y_{t}}{y_{t}\left(\mu=M_{Z}\right)}, \quad L=\frac{\lambda}{\lambda\left(\mu=M_{Z}\right)}
$$

and get

$$
\begin{aligned}
\left.\beta_{\lambda}\right|_{\mu=M_{Z}}= & (\underbrace{-1.7 Y^{4}}_{1 \text { loop }}) 10^{-2}+(\underbrace{5.0 L Y^{2}}_{1 \text { loop }} \underbrace{+1.5 L^{2}}_{1 \text { loop }}) 10^{-3} \\
& +(\underbrace{-8.5 G^{2} Y^{4}}_{2 \text { loop }} \underbrace{+5.0 Y^{6}}_{2 \text { loop }} \underbrace{+3.1 G^{2} L Y^{2}}_{2 \text { loop }}) 10^{-4} \\
& +(\underbrace{7.9 G^{2} Y^{6}}_{3 \text { loop }} \underbrace{-4.8 Y^{8}}_{3 \text { loop }} \underbrace{-5.3 L^{2} Y^{2}}_{2 \text { loop }} \underbrace{-3.1 G^{2} L Y^{4}}_{3 \text { loop }} \\
& \underbrace{-2.5 G^{4} Y^{4}}_{3 \text { loop }} \underbrace{+2.6 G^{4} L Y^{2}}_{3 \text { loop }} \underbrace{-1.7 L^{3}}_{2 \text { loop }}) 10^{-5} \\
& +(\underbrace{-7.5 L Y^{4}}_{2 \text { loop }} \underbrace{+7.8 L^{2} Y^{4}}_{3 \text { loop }} \underbrace{-6.6 L Y^{6}}_{3 \text { loop }} \underbrace{+1.1 G^{2} L^{2} Y^{2}}_{3 \text { loop }}) 10^{-6} \\
& +(\underbrace{5.7 L^{3} Y^{2}}_{3 \text { loop }} \underbrace{+5.9 L^{4}}_{3 \text { loop }}) 10^{-7} .
\end{aligned}
$$

We see that the decrease of the effective four $-\Phi$ coupling with increasing energy is induced by top quark loops. Without quarks there would be an increase. It is also worth noting that the individual contributions at three-loop level are much larger than the overall effect due to huge cancellations. Consider for example the five numerically largest three-loop terms at $\mu=M_{Z}$ :

$$
\left(7.9 G^{2} Y^{6}-4.8 Y^{8}-3.1 G^{2} L Y^{4}-2.5 G^{4} Y^{4}+2.6 G^{4} L Y^{2}\right) 10^{-5} .
$$

The total contribution from these terms is by almost two orders of magnitude smaller than the size of the largest one. 
For the top-Yukawa $\beta$-functions we find

$$
\begin{aligned}
\frac{\beta_{y_{t}}^{(1)}}{y_{t}}= & \frac{9}{4} y_{t}^{2}-4 g_{s}^{2}, \\
\frac{\beta_{y_{t}}^{(2)}}{y_{t}}= & 3 \lambda^{2}-6 y_{t}^{2} \lambda-6 y_{t}^{4}+18 g_{s}^{2} y_{t}^{2}+g_{s}^{4}\left(-\frac{202}{3}+\frac{20}{9} n_{f}\right), \\
\frac{\beta_{y_{t}}^{(3)}}{y_{t}}= & -18 \lambda^{3}+\frac{15}{8} y_{t}^{2} \lambda^{2}+99 y_{t}^{4} \lambda+y_{t}^{6}\left(\frac{339}{16}+\frac{27}{4} \zeta_{3}\right) \\
& +8 g_{s}^{2} y_{t}^{2} \lambda-\frac{157}{2} g_{s}^{2} y_{t}^{4}+g_{s}^{4} y_{t}^{2}\left(\frac{4799}{12}-\frac{27}{2} n_{f}-114 \zeta_{3}\right) \\
& +g_{s}^{6}\left(-1249+\frac{2216}{27} n_{f}+\frac{140}{81} n_{f}^{2}+\frac{160}{3} \zeta_{3} n_{f}\right) .
\end{aligned}
$$

As has already been mentioned above the $\lambda$-corrections are negligible here. Evaluating $\beta_{y_{t}}$ at the scale $\mu=M_{Z}$ (with an assumed Higgs mass of $125 \mathrm{GeV}$ and $n_{f}=6$ ) we get a value of $\sim(-0.023)$ at one-loop level which means a decrease of $y_{t}$ and therefore the top mass with increasing energy. This is due to the QCD corrections. In the absence of QCD the opposite would be the case as we can see from the term $\propto y_{t}^{2}$ in $\frac{\beta_{y_{t}}}{y_{t}}$. The two and three-loop corrections are $\sim 16.6 \%$ and $\sim 0.7 \%$ with respect to the one-loop result and so quite high compared e.g. to the case of $\beta_{g_{s}}$ discussed below. We use again the labels (27) to get an impression of the individual terms:

$$
\begin{aligned}
\left.\beta_{y_{t}}\right|_{\mu=M_{Z}}= & (\underbrace{-3.7 Y G^{2}}_{1 \text { loop }} \underbrace{+1.3 Y^{3}}_{1 \text { loop }}) 10^{-2}+(\underbrace{-4.7 Y G^{4}}_{2 \text { loop }}) 10^{-3} \\
& +(\underbrace{9.8 Y^{3} G^{2}}_{2 \text { loop }} \underbrace{-2.5 Y G^{6}}_{3 \text { loop }} \underbrace{-2.1 Y^{5}}_{2 \text { loop }}) 10^{-4} \\
& +(\underbrace{9.3 Y^{3} G^{4}}_{3 \text { loop }} \underbrace{-3.1 L Y^{3}}_{2 \text { loop }} \underbrace{-2.5 Y^{5} G^{2}}_{3 \text { loop }}) 10^{-5} \\
& +(\underbrace{6.0 Y^{7}}_{3 \text { loop }} \underbrace{+3.0 L Y^{5}}_{3 \text { loop }} \underbrace{+2.3 L^{2} Y}_{2 \text { loop }}) 10^{-6} \\
& +(\underbrace{3.9 L Y^{3} G^{2}}_{3 \text { loop }}) 10^{-7}+(\underbrace{-1.2 L^{3} Y}_{3 \text { loop }}) 10^{-8}+(\underbrace{8.5 L^{2} Y^{3}}_{3 \text { loop }}) 10^{-9} .
\end{aligned}
$$

For the strong coupling we get

$$
\begin{aligned}
& \frac{\beta_{g_{s}}^{(1)}}{g_{s}}=g_{s}^{2}\left(-\frac{11}{2}+\frac{1}{3} n_{f}\right), \\
& \frac{\beta_{g_{s}}^{(2)}}{g_{s}}=-g_{s}^{2} y_{t}^{2}+g_{s}^{4}\left(-51+\frac{19}{3} n_{f}\right), \\
& \frac{\beta_{g_{s}}^{(3)}}{g_{s}}=\frac{15}{2} g_{s}^{2} y_{t}^{4}-20 g_{s}^{4} y_{t}^{2}+g_{s}^{6}\left(-\frac{2857}{4}+\frac{5033}{36} n_{f}-\frac{325}{108} n_{f}^{2}\right) .
\end{aligned}
$$

In order to numerically compare the higher order corrections to the above $\beta$-functions we also give the evaluation of $\beta_{g_{s}}$ at the scale $\mu=M_{Z}$ and with $n_{f}=6$. The one-loop contribution 
is $\sim(-0.04)$ to which the two and three-loop calculations give corrections of $\sim 3.7 \%$ and $\sim(-0.02) \%$ respectively. Very small $\lambda$-corrections to $\beta_{g_{s}}$ do not appear until four loops. With eq. (27) and the above assumptions we get

$$
\begin{aligned}
\left.\beta_{g_{s}}\right|_{\mu=M_{Z}}= & (\underbrace{-4.0 G^{3}}_{1 \text { loop }}) 10^{-2}+(\underbrace{-1.4 G^{5}}_{2 \text { loop }}) 10^{-3} \\
& +(\underbrace{-6.9 Y^{2} G^{3}}_{2 \text { loop }} \underbrace{+1.7 G^{7}}_{3 \text { loop }} \underbrace{-1.3 Y^{2} G^{5}}_{3 \text { loop }}) 10^{-5}+(\underbrace{3.1 Y^{4} G^{3}}_{3 \text { loop }}) 10^{-6} .
\end{aligned}
$$

The running of the $m^{2}$ parameter is given by

$$
\begin{aligned}
\frac{\beta_{m^{2}}^{(1)}}{m^{2}}= & 6 \lambda+3 y_{t}^{2}, \\
\frac{\beta_{m^{2}}^{(2)}}{m^{2}}= & -30 \lambda^{2}-36 y_{t}^{2} \lambda-\frac{27}{4} y_{t}^{4}+20 g_{s}^{2} y_{t}^{2}, \\
\frac{\beta_{m^{2}}^{(3)}}{m^{2}}= & 1026 \lambda^{3}+\frac{297}{2} y_{t}^{2} \lambda^{2}+y_{t}^{4} \lambda\left(\frac{351}{4}+216 \zeta_{3}\right) \\
& +y_{t}^{6}\left(\frac{1605}{16}+45 \zeta_{3}\right)+g_{s}^{2} y_{t}^{2} \lambda\left(-612+576 \zeta_{3}\right) \\
& +g_{s}^{2} y_{t}^{4}\left(\frac{447}{2}-360 \zeta_{3}\right)+g_{s}^{4} y_{t}^{2}\left(\frac{910}{3}-16 n_{f}-24 \zeta_{3}\right) .
\end{aligned}
$$

Again we evaluate $\beta_{m^{2}}$ at the scale $\mu=M_{Z}$ (with an assumed Higgs mass of $125 \mathrm{GeV}$ and $\left.n_{f}=6\right)$ and get a value of $\sim(+0.023)$ at one-loop level which means an increase of $m^{2}$ at higher energy scales. The two and three-loop corrections are $\sim 2.9 \%$ and $\sim 0.32 \%$. With the labels (27) we can estimate the contributions of the individual terms:

$$
\begin{aligned}
\left.\frac{\beta_{m^{2}}}{m^{2}}\right|_{\mu=M_{Z}}= & (\underbrace{1.8 Y^{2}}_{1 \text { loop }}) 10^{-2}+(\underbrace{5.3 L}_{1 \text { loop }} \underbrace{+1.1 G^{2} Y^{2}}_{2 \text { loop }}) 10^{-3}+(\underbrace{-2.4 Y^{4}}_{2 \text { loop }} \underbrace{-1.9 L Y^{2}}_{2 \text { loop }}) 10^{-4} \\
& +(\underbrace{9.4 G^{4} Y^{2}}_{3 \text { loop }} \underbrace{-7.0 G^{2} Y^{4}}_{3 \text { loop }} \underbrace{+3.3 Y^{6}}_{3 \text { loop }} \underbrace{-2.4 L^{2}}_{2 \text { loop }} \underbrace{+1.1 L Y^{4}}_{3 \text { loop }}) 10^{-5} \\
& +(\underbrace{4.0 G^{2} L Y^{2}}_{3 \text { loop }}) 10^{-6}+(\underbrace{7.1 L^{3}}_{3 \text { loop }} \underbrace{+7.0 L^{2} Y^{2}}_{3 \text { loop }}) 10^{-7} .
\end{aligned}
$$

\section{Results for the anomalous dimensions}

In this section we give the anomalous dimensions of the physical fields in this setup. Note that because of the $\mathrm{SU}(2)$ symmetry $\Phi_{1}$ and $\Phi_{2}$ must have the same anomalous dimension $\gamma_{2}^{\Phi}$. The same holds for the left-handed part of top and bottom quarks: $\gamma_{2, L}^{t}=\gamma_{2, L}^{b}$. For the quark flavours $q$ which do not participate in the Yukawa interaction there is no difference between the left- and right-handed part as they are renormalized by the same Z-factor: $\gamma_{2}^{q} \equiv \gamma_{2, L}^{q}=\gamma_{2, R}^{q}$. This also applies to the right-handed part of the bottom quark: $\gamma_{2, R}^{b}=\gamma_{2}^{q}$. All these relations have been tested explicitly during our calculation which provides a nice additional check. 


$$
\begin{aligned}
\gamma_{2}^{q(1)}= & g_{s}^{2} C_{F}(1-\xi), \\
\gamma_{2}^{q(2)}= & g_{s}^{4}\left(-\frac{3}{2} C_{F}^{2}+\frac{17}{2} C_{A} C_{F}-2 n_{f} T_{F} C_{F}-\frac{5}{2} \xi C_{A} C_{F}+\frac{1}{4} \xi^{2} C_{A} C_{F}\right), \\
\gamma_{2}^{q(3)}= & 6 T_{F} C_{F} g_{s}^{4} y_{t}^{2}+g_{s}^{6}\left(\frac{3}{2} C_{F}^{3}-\frac{143}{4} C_{A} C_{F}^{2}+\frac{10559}{144} C_{A}^{2} C_{F}+3 n_{f} T_{F} C_{F}^{2}\right. \\
& -\frac{1301}{36} n_{f} C_{A} T_{F} C_{F}+\frac{20}{9} n_{f}^{2} T_{F}^{2} C_{F}+12 \zeta_{3} C_{A} C_{F}^{2}-\frac{15}{2} \zeta_{3} C_{A}^{2} C_{F}-\frac{371}{32} \xi C_{A}^{2} C_{F} \\
& \left.+\frac{17}{4} \xi n_{f} C_{A} T_{F} C_{F}-\frac{3}{2} \xi \zeta_{3} C_{A}^{2} C_{F}+\frac{69}{32} \xi^{2} C_{A}^{2} C_{F}+\frac{3}{8} \xi^{2} \zeta_{3} C_{A}^{2} C_{F}-\frac{5}{16} \xi^{3} C_{A}^{2} C_{F}\right) .
\end{aligned}
$$

For $y_{t}=0$ this is in agreement with the well-known QCD result [25]. The renormalization constants for t,b and q can also be found in [23] up to order $g_{s}^{6}$ and $g_{s}^{4} y_{t}^{2}$ where the calculation has been performed in the broken phase of the SM.

$$
\begin{aligned}
\gamma_{2, L}^{t(1)}= & \gamma_{2}^{q(1)}+\frac{1}{2} y_{t}^{2}, \\
\gamma_{2, L}^{t(2)}= & \gamma_{2}^{q(2)}-y_{t}^{4}\left(\frac{1}{4}+\frac{3}{4} d_{R}\right)-2 C_{F} g_{s}^{2} y_{t}^{2} \\
\gamma_{2, L}^{t(3)}= & \gamma_{2}^{q(3)}-\frac{33}{4} y_{t}^{2} \lambda^{2}+6 y_{t}^{4} \lambda+y_{t}^{6}\left(-\frac{3}{2}+\frac{29}{8} d_{R}-\frac{3}{8} d_{R}^{2}+\frac{3}{2} \zeta_{3}\right) \\
& +g_{s}^{2} y_{t}^{4}\left(\frac{13}{2} C_{F}+\frac{5}{8} C_{F} d_{R}+6 \zeta_{3} C_{F} d_{R}\right) \\
& +g_{s}^{4} y_{t}^{2}\left(-\frac{51}{8} C_{F}^{2}+\frac{31}{8} C_{A} C_{F}-\frac{3}{2} n_{f} T_{F} C_{F}+6 \zeta_{3} C_{F}^{2}-15 \zeta_{3} C_{A} C_{F}\right) . \\
\gamma_{2, R}^{t(1)}= & \gamma_{2}^{q(1)}+y_{t}^{2}, \\
\gamma_{2, R}^{t(2)}= & \gamma_{2}^{q(2)}-y_{t}^{4}\left(\frac{1}{4}+\frac{3}{2} d_{R}\right)-4 C_{F} g_{s}^{2} y_{t}^{2}, \\
\gamma_{2, R}^{t(3)}= & \gamma_{2}^{q(3)}-\frac{33}{2} y_{t}^{2} \lambda^{2}+12 y_{t}^{4} \lambda+y_{t}^{6}\left(-\frac{33}{16}+\frac{53}{8} d_{R}-\frac{3}{4} d_{R}^{2}+3 \zeta_{3}\right) \\
& +g_{s}^{2} y_{t}^{4}\left(5 C_{F}+\frac{5}{4} C_{F} d_{R}+12 \zeta_{3} C_{F} d_{R}\right) \\
& +g_{s}^{4} y_{t}^{2}\left(-\frac{51}{4} C_{F}^{2}+\frac{31}{4} C_{A} C_{F}-3 n_{f} T_{F} C_{F}+12 \zeta_{3} C_{F}^{2}-30 \zeta_{3} C_{A} C_{F}\right) .
\end{aligned}
$$

$$
\begin{aligned}
\gamma_{2}^{\Phi(1)}= & d_{R} y_{t}^{2}, \\
\gamma_{2}^{\Phi(2)}= & 6 \lambda^{2}-\frac{9}{4} d_{R} y_{t}^{4}+5 C_{F} d_{R} g_{s}^{2} y_{t}^{2}, \\
\gamma_{2}^{\Phi(3)}= & -36 \lambda^{3}-\frac{45}{2} d_{R} y_{t}^{2} \lambda^{2}+15 d_{R} y_{t}^{4} \lambda \\
& +y_{t}^{6}\left(-\frac{25}{16} d_{R}+6 d_{R}^{2}+3 \zeta_{3} d_{R}\right)+g_{s}^{2} y_{t}^{4}\left(\frac{15}{8} C_{F} d_{R}-18 \zeta_{3} C_{F} d_{R}\right) \\
& +g_{s}^{4} y_{t}^{2}\left(-\frac{119}{4} C_{F}^{2} d_{R}+\frac{77}{2} C_{A} C_{F} d_{R}-8 n_{f} T_{F} C_{F} d_{R}+36 \zeta_{3} C_{F}^{2} d_{R}-18 \zeta_{3} C_{A} C_{F} d_{R}\right),
\end{aligned}
$$


The purely $\lambda$-dependent part of this has been computed before in [18, 19].

$$
\begin{aligned}
\gamma_{2}^{g(1)}= & g_{s}^{2}\left(-\frac{5}{3} C_{A}+\frac{4}{3} n_{f} T_{F}-\frac{1}{2} \xi C_{A}\right), \\
\gamma_{2}^{g(2)}= & -4 T_{F} g_{s}^{2} y_{t}^{2}+g_{s}^{4}\left(-\frac{23}{4} C_{A}^{2}+4 n_{f} T_{F} C_{F}+5 n_{f} C_{A} T_{F}-\frac{15}{8} \xi C_{A}^{2}+\frac{1}{4} \xi^{2} C_{A}^{2}\right), \\
\gamma_{2}^{g(3)}= & g_{s}^{2} y_{t}^{4}\left(9 T_{F}+7 T_{F} d_{R}\right)-g_{s}^{4} y_{t}^{2}\left(6 T_{F} C_{F}+\frac{25}{2} C_{A} T_{F}\right) \\
& +g_{s}^{6}\left(-\frac{4051}{144} C_{A}^{3}-2 n_{f} T_{F} C_{F}^{2}+\frac{5}{18} n_{f} C_{A} T_{F} C_{F}+\frac{875}{18} n_{f} C_{A}^{2} T_{F}-\frac{44}{9} n_{f}^{2} T_{F}^{2} C_{F}\right. \\
& -\frac{76}{9} n_{f}^{2} C_{A} T_{F}^{2}+\frac{3}{2} \zeta_{3} C_{A}^{3}+24 \zeta_{3} n_{f} C_{A} T_{F} C_{F}-18 \zeta_{3} n_{f} C_{A}^{2} T_{F}-\frac{127}{16} \xi C_{A}^{3} \\
& \left.+2 \xi n_{f} C_{A}^{2} T_{F}-\frac{9}{8} \xi \zeta_{3} C_{A}^{3}+\frac{27}{16} \xi^{2} C_{A}^{3}+\frac{3}{16} \xi^{2} \zeta_{3} C_{A}^{3}-\frac{7}{32} \xi^{3} C_{A}^{3}\right) .
\end{aligned}
$$

This is also in agreement with [25] for $y_{t}=0$ and with [24] for $y_{t}=0, \xi=0$. For $d_{R}=3$ and $T_{F}=\frac{1}{2}(\mathrm{QCD})$ these results are as follows:

$$
\begin{aligned}
\gamma_{2}^{q(1)}= & \frac{4}{3}(1-\xi) g_{s}^{2}, \\
\gamma_{2}^{q(2)}= & g_{s}^{4}\left(\frac{94}{3}-\frac{4}{3} n_{f}-10 \xi+\xi^{2}\right), \\
\gamma_{2}^{q(3)}= & 4 g_{s}^{4} y_{t}^{2}+g_{s}^{6}\left(+\frac{24941}{36}-\frac{1253}{18} n_{f}+\frac{20}{27} n_{f}^{2}-26 \zeta_{3}-\frac{1113}{8} \xi\right. \\
+ & \left.\frac{17}{2} \xi n_{f}-18 \xi \zeta_{3}+\frac{207}{8} \xi^{2}+\frac{9}{2} \xi^{2} \zeta_{3}-\frac{15}{4} \xi^{3}\right), \\
\gamma_{2, L}^{t(1)}= & \gamma_{2}^{q(1)}+\frac{1}{2} y_{t}^{2}, \\
\gamma_{2, L}^{t(2)}= & \gamma_{2}^{q(2)}-\frac{5}{2} y_{t}^{4}-\frac{8}{3} g_{s}^{2} y_{t}^{2}, \\
\gamma_{2, L}^{t(3)}= & \gamma_{2}^{q(3)}-\frac{33}{4} y_{t}^{2} \lambda^{2}+6 y_{t}^{4} \lambda+y_{t}^{6}\left(6+\frac{3}{2} \zeta_{3}\right) \\
& +g_{s}^{2} y_{t}^{4}\left(\frac{67}{6}+24 \zeta_{3}\right)+g_{s}^{4} y_{t}^{2}\left(\frac{25}{6}-n_{f}-\frac{148}{3} \zeta_{3}\right) . \\
& +g_{s}^{2} y_{t}^{4}\left(\frac{15}{2}-72 \zeta_{3}\right)+g_{s}^{4} y_{t}^{2}\left(\frac{910}{3}-16 n_{f}-24 \zeta_{3}\right), \\
\gamma_{2, R}^{t(1)}= & \gamma_{2}^{q(1)}+y_{t}^{2}, \\
\gamma_{2, R}^{t(2)}= & \gamma_{2}^{q(2)}-\frac{19}{4} y_{t}^{4}-\frac{16}{3} g_{s}^{2} y_{t}^{2}, \\
\gamma_{2, R}^{t(3)}= & \gamma_{2}^{q(3)}-\frac{33}{2} y_{t}^{2} \lambda^{2}+12 y_{t}^{4} \lambda+y_{t}^{6}\left(\frac{177}{16}+3 \zeta_{3}\right) \\
& +g_{s}^{2} y_{t}^{4}\left(\frac{35}{3}+48 \zeta_{3}\right)+g_{s}^{4} y_{t}^{2}\left(\frac{25}{3}-2 n_{f}-\frac{296}{3} \zeta_{3}\right), \\
\gamma_{2}^{\Phi(2)}= & 6 \lambda^{2}-\frac{27}{4} y_{t}^{4}+20 g_{s}^{2} y_{t}^{2}, \\
\gamma_{2}^{\Phi(1)}= & -36 \lambda_{t}^{2}, \\
\gamma_{2}(3) & \frac{135}{2} y_{t}^{2} \lambda^{2}+45 y_{t}^{4} \lambda+y_{t}^{6}\left(\frac{789}{16}+9 \zeta_{3}\right) \\
& \\
&
\end{aligned}
$$




$$
\begin{aligned}
\gamma_{2}^{g(1)}= & g_{s}^{2}\left(-5+\frac{2}{3} n_{f}-\frac{3}{2} \xi\right), \\
\gamma_{2}^{g(2)}= & -2 g_{s}^{2} y_{t}^{2}+g_{s}^{4}\left(-\frac{207}{4}+\frac{61}{6} n_{f}-\frac{135}{8} \xi+\frac{9}{4} \xi^{2}\right), \\
\gamma_{2}^{g(3)}= & 15 g_{s}^{2} y_{t}^{4}-\frac{91}{4} g_{s}^{4} y_{t}^{2} \\
& +g_{s}^{6}\left(-\frac{12153}{16}+\frac{7831}{36} n_{f}-\frac{215}{27} n_{f}^{2}+\frac{81}{2} \zeta_{3}-33 \zeta_{3} n_{f}-\frac{3429}{16} \xi\right. \\
& \left.+9 \xi n_{f}-\frac{243}{8} \xi \zeta_{3}+\frac{729}{16} \xi^{2}+\frac{81}{16} \xi^{2} \zeta_{3}-\frac{189}{32} \xi^{3}\right) .
\end{aligned}
$$

\section{The evolution of the quartic Higgs coupling}

The quartic Higgs coupling $\lambda$ is of special interest as it is directly related to the Higgs mass $M_{H}$. If we assume that the SM is valid up to some high energy scale $\Lambda$, then the value of $M_{H}$ should meet the constraints

$$
m_{\min }<M_{H}<m_{\max } .
$$

Here the upper limit is related to the well-known fact that the running Higgs self-coupling develops a Landau pole8 if $M_{H}$ is large [33 35]. For $\Lambda=M_{\text {Planck }}=10^{18} \mathrm{GeV}$ the estimated value of $m_{\max }$ is around $175 \mathrm{GeV}$ [33 36], which is already excluded by experiments carried out at the LHC and the Tevatron.

The lower limit $m_{\min }$ follows from the requirement of the vacuum stability [37 39 ]. In order to find $m_{\min }$ one should construct the effective Higgs potential $V[\phi]$ including radiative corrections and sum possible large logarithms using the standard method of the Renormalization Group (for a review see, e.g. [40]). Once this has been done, the condition that the potential $V[\phi]$ does not develop a deeper minimum in addition to the standard one for all values of $\phi<\Lambda$ fixes $m_{\min }$.

In our analysis we will use a simplified approach for finding $m_{\min }$, namely the requirement that the running coupling constant $\lambda(\mu)$ stay non-negative for all $\mu$ less than $\Lambda$. It has been shown in [34,41] that the simplified approach is essentially equivalent to the one based on the use of the effective potential provided the instabilty of $V[\phi]$ can only happen at $\phi \gg M_{Z}$.

In this section we investigate the effect of the three-loop result $\beta_{\lambda}^{(3)}$ on the running of $\lambda$ and therefore its effect on the stability of the electroweak vacuum in the SM. For this we also include the electroweak contributions up to the two-loop level. The two-loop $\beta$-functions for the SM gauge couplings have been derived in [28 31]. The two-loop results for the Yukawacouplings and $\lambda$ can be found in [20 22]. Now we add the three-loop results derived in the previous section and investigate the effect this has on the evolution of our couplings (for a recent similar analysis, using the two-loop running, see, e.g. [9, 42,43]).

To find starting values for the running of the couplings we should account for the fact that the physical parameters (e.g. pole masses) are related to the ones in the $\overline{\mathrm{MS}}$-scheme in a non-trivial way (see e.g. [44-46]). For example eq. (15) is only valid at tree level. For the higher order corrections we take the electroweak ones at one-loop and the QCD ones at twoloop level from [44,45]. These matching relations depend on the exact values of $\alpha_{s}\left(M_{Z}\right)$ and

\footnotetext{
${ }^{8}$ This is true in the one-loop approximation. At two loops the Landau pole is replaced by an ultraviolet metastable fixed point with the resulting fixed point value of $\lambda$ being outside the weak coupling region.
} 
the pole mass $M_{t}$ of the top quark and of course the mass of the Higgs boson $M_{H}$. For the latter we consider the cases $M_{H}=124 \mathrm{GeV}$ and $M_{H}=126 \mathrm{GeV}$. For the other two we use the values

$$
\alpha_{s}\left(M_{Z}\right)=0.1184 \pm 0.0007 \quad \text { and } \quad M_{t}=172.9 \pm 0.6 \pm 0.9 \mathrm{GeV} \quad \text { [47]. }
$$

One should also keep in mind that the matching relations themselves receive contributions from not yet known higher order corrections. The corresponding uncertainty in $m_{m i n}$ has been estimated in [4,42] and found to be about $2 \mathrm{GeV}$.

Fig. [6 shows the evolution of $\lambda$ in this framework up to the Planck scale. To estimate the dependence of the $\lambda$-running on the parameters $\alpha_{s}\left(M_{Z}\right)$ and $M_{t}$ we give the shifted curves for $\lambda(\mu)$ when we change these parameters by $\pm \sigma$ as given in eq. (45) 9 As the two- and three-loop curves are very close together we zoom in on the region where $\lambda$ crosses over to negative values in Fig. 7. In this plot we give the $\alpha_{s}$-uncertainty for the two- and three-loop curves to compare between this uncertainty and the shift from two to three loops. Note that there is a considerable difference between $M_{H}=124 \mathrm{GeV}$ and $M_{H}=126 \mathrm{GeV}$ which means that the evolution of $\lambda$ is very sensitive to the value of the Higgs mass. Given a fixed value for $M_{H}$ the largest uncertainty lies in the exact value of the top mass. The second largest uncertainty comes from $\alpha_{s}$. The total effect due to the three-loop part of the $\beta$-functions is somewhat smaller than latter as can be seen best in Fig. 7. Still, it is worthy of note that the three-loop corrections to the $\beta$-functions presented here enhance the stability of the SM electroweak vacuum.

The smallness of the three-loop correction to $\beta_{\lambda}$ seems to be somewhat coincidental as the aforementioned cancellations of individual terms in $\beta_{\lambda}^{(3)}$ depend strongly on the value of $M_{H}$. Finding a Higgs with a mass of 124 to $126 \mathrm{GeV}$ would therefore mean an excellent convergence of the perturbation series for $\beta_{\lambda}$. Another intriguing consequence of a Higgs mass in that region is the uncertainty whether $\lambda$ becomes indeed negative at high scales or not. If we take e.g. $M_{H}=126 \mathrm{GeV}$ and $\alpha_{s}=0.1184$ and decreas 10 the top mass from $M_{t}=172.9 \mathrm{GeV}$ to $M_{t}=171.25 \mathrm{GeV}\left(M_{t}=171.16 \mathrm{GeV}\right.$ without the three-loop corrections), then $\lambda$ stays positive up to the Planck scale $M_{\text {Planck }}$ in our framework. The same effect can be achieved for $M_{H}=126 \mathrm{GeV}$ and $M_{t}=172.9 \mathrm{GeV}$ by increasing $\alpha_{s}=0.1184$ by $6.5 \sigma_{\alpha_{s}}\left(7 \sigma_{\alpha_{s}}\right.$ without the three-loop corretions). A combined scenario for $M_{H}=126 \mathrm{GeV}$ would be a shift of $M_{t}=172.9 \mathrm{GeV}$ by $-1 \sigma_{M_{t}}=-1.5 \mathrm{GeV}$ and of $\alpha_{s}=0.1184$ by $+1 \sigma_{\alpha_{s}}=+0.0007 \mathrm{GeV}$ which would also make $\lambda$ positive up to the Planck scale.

Thus, we conclude that at present no definite answer can be given to the question whether the SM vacuum is stable all the way up to the Planck scale or not. If indeed a SM Higgs boson is found with a mass of 124 to $126 \mathrm{GeV}$, this is a good motivation for determining $\alpha_{s}\left(M_{Z}\right)$ and $M_{t}$ as accurately as possible as well as calculating the SM $\beta$-functions to the highest achievable accuracy.

\section{Conclusions}

We have computed the three-loop corrections to the evolution the top-Yukawa coupling, the strong coupling and the quartic Higgs self-coupling in the unbroken SM with the numerically small gauge coupling constants $g_{1}$ and $g_{2}$ and all Yukawa couplings except for $y_{t}$ set to zero.

\footnotetext{
${ }^{9}$ In order not to make the plot too crowded these shifted curves are only given for the two-loop result. The difference to the three-loop result is similar to the one between the two- and three-loop curves for $\alpha_{s}\left(M_{Z}\right)=0.1184$ and $M_{t}=172.9 \mathrm{GeV}$.

${ }^{10}$ Smaller values for $M_{t}$ or larger values for $\alpha_{s}$ increase the stability of the vacuum.
} 

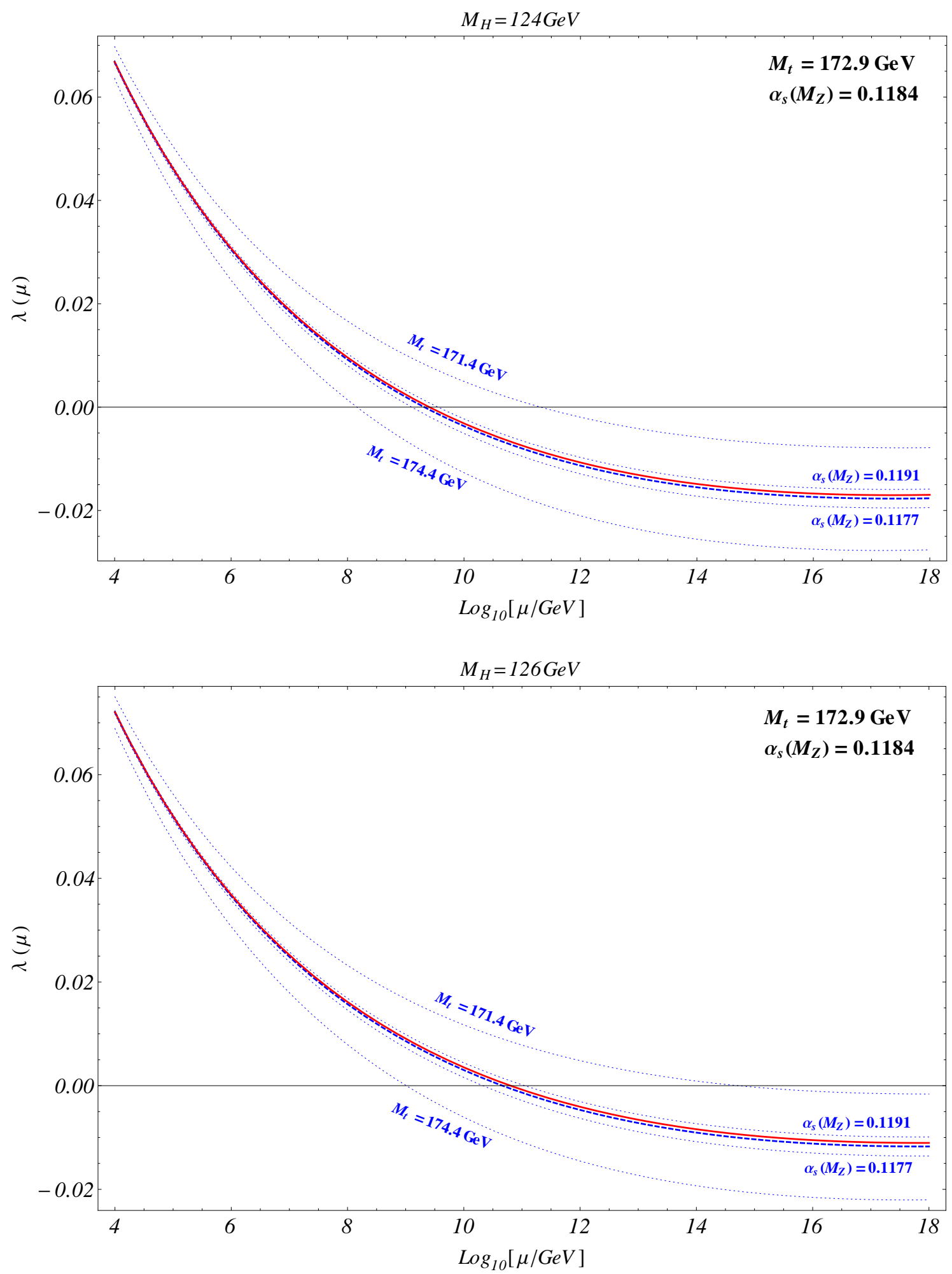

Figure 6: Evolution of $\lambda$ with the scale $\mu$ : 2 loop (dashed, blue) and 3 loop (continuous, red) results; Uncertainties with respect to the two-loop result: $\pm 1 \sigma_{\alpha_{s}}, \pm 1 \sigma_{M_{t}}$ (dotted)

The implications of our calculation on the stability of the electroweak vacuum in the SM can be summarized as follows:

- The total effect of the three-loop terms is relatively small which is not self-evident as 

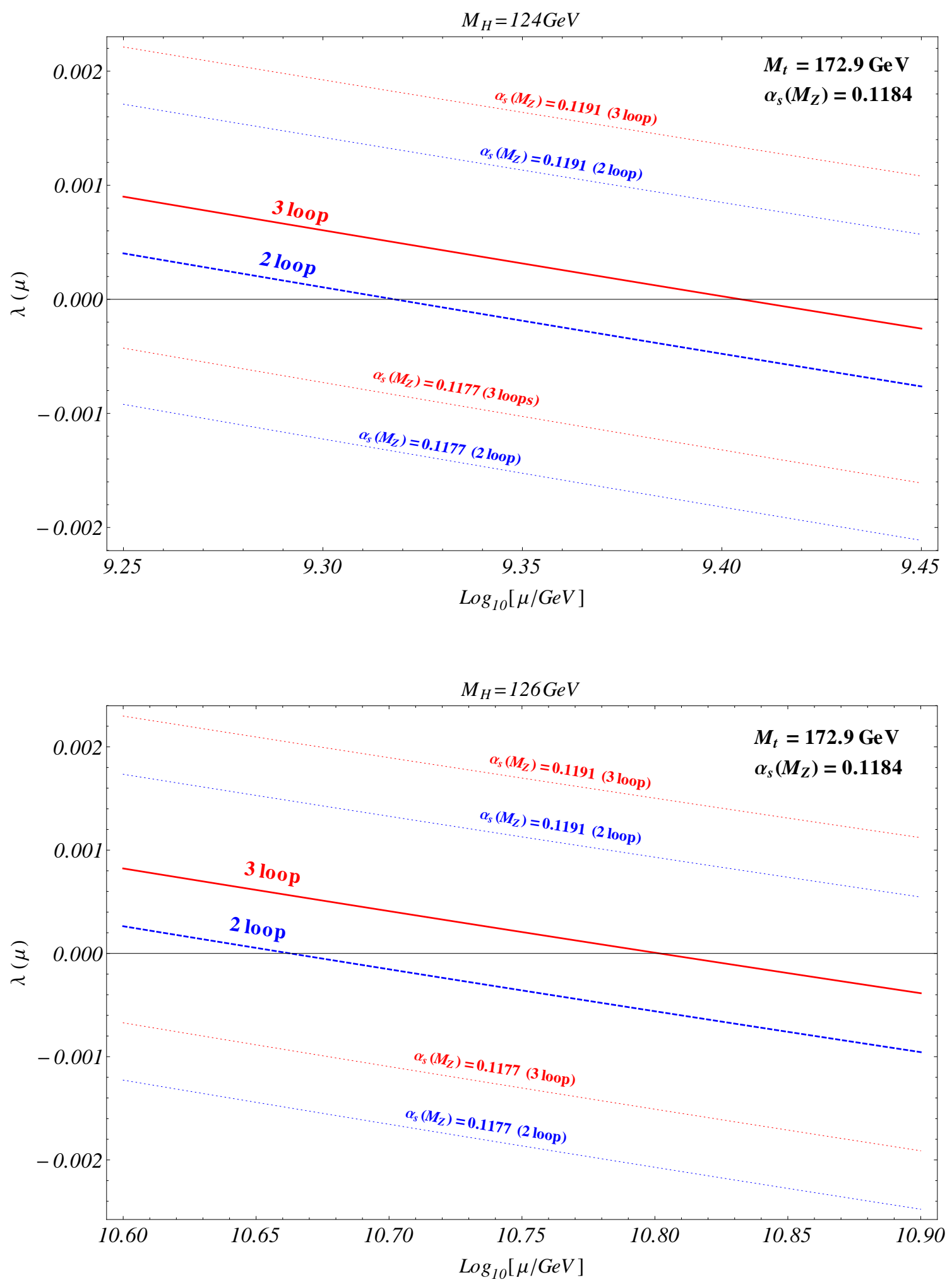

Figure 7: Evolution of $\lambda$ with the scale $\mu$ : 2 loop (dashed, blue) and 3 loop (continuous, red) results; Uncertainties with respect to the two- and three-loop results: $\pm 1 \sigma_{\alpha_{s}}$ (dotted)

the individual terms in $\beta_{\lambda}^{(3)}$ are much larger than the final value due to significant cancellations for a Higgs mass in the vicinity of $125 \mathrm{GeV}$. 
- The evolution of $\lambda$ is very sensitive to the values of the Higgs mass, the top mass and $\alpha_{s}\left(M_{Z}\right)$. If we take e.g. $M_{H}=126 \mathrm{GeV}$ and decrease the top mass by about $1.7 \mathrm{GeV}$, then $\lambda$ stays positive up to the Planck scale (a similar observation has been made in [42]). This is a very good motivation for high precision measurements of $\alpha_{s}\left(M_{Z}\right)$ and $M_{t}$. With the latter values known more precisely, the account of the the threeloop effects in the evolution of the quartic Higgs self-coupling would be essential in considering the problem of the stability of the electroweak vacuum in the SM.

- In this context it may also be useful to calculate both the electroweak contributions to $\beta_{\lambda}^{(3)}$ and $\beta_{y_{t}}^{(3)}$ at three-loop level as well as the matching of experimentally measurable on-shell parameters and $\overline{\mathrm{MS}}$-parameters to a higher accuracy.

We thank Luminita Mihaila, Jens Salomon and Matthias Steinhauser for useful discussions and informing us on the results of 32 before their publication. We thank Johann Kühn for valuable comments and support. Last but not least, we are grateful to Fedor Bezrukov and Mikhail Shaposhnikov for gently drawing our attention to the subject, numerous discussions and providing us with their version of a Mathematica package to perform one-loop matching in the SM.

In conclusion we want to mention that all our calculations have been performed on a SGI ALTIX 24-node IB-interconnected cluster of 8-cores Xeon computers using the thread-based [48] version of FORM [10]. The Feynman diagrams have been drawn with the Latex package Axodraw [49].

This work has been supported by the Deutsche Forschungsgemeinschaft in the Sonderforschungsbereich/Transregio SFB/TR-9 "Computational Particle Physics".

Note added. More elaborated analyses of the vacuum stability which take into account the three-loop running as obtained here and two-loop matching corrections can be found in recent works [50 53$]$.

\section{References}

[1] F. L. Bezrukov and M. Shaposhnikov, The Standard Model Higgs boson as the inflaton. Phys. Lett. B659 (2008) 703-706, arXiv:0710.3755 [hep-th].

[2] F. L. Bezrukov, A. Magnin, and M. Shaposhnikov, Standard Model Higgs boson mass from inflation. Phys. Lett. B675 (2009) 88-92, arXiv:0812.4950 [hep-ph].

[3] A. De Simone, M. P. Hertzberg, and F. Wilczek, Running Inflation in the Standard Model. Phys. Lett. B678 (2009) 1-8, arXiv:0812.4946 [hep-ph].

[4] F. Bezrukov and M. Shaposhnikov, Standard Model Higgs boson mass from inflation: two loop analysis. JHEP 07 (2009) 089, arXiv:0904.1537 [hep-ph].

[5] M. Shaposhnikov and C. Wetterich, Asymptotic safety of gravity and the Higgs boson mass. Phys. Lett. B683 (2010) 196-200, arXiv:0912.0208 [hep-th].

[6] ATLAS Collaboration, G. Aad et al., Combined search for the Standard Model Higgs boson using up to $4.9 \mathrm{fb}-1$ of pp collision data at sqrt(s) $=7 \mathrm{TeV}$ with the ATLAS detector at the LHC. Phys. Lett. B710 (2012) 49-66, arXiv:1202.1408 [hep-ex].

[7] CMS Collaboration, S. Chatrchyan et al., Combined results of searches for the standard model Higgs boson in pp collisions at sqrt(s) $=7 \mathrm{TeV}$. arXiv:1202.1488 [hep-ex]. 
[8] TEVNPH (Tevatron New Phenomina and Higgs Working Group)

Collaboration, Combined CDF and DO Search for Standard Model Higgs Boson

Production with up to $10.0 \mathrm{fb}-1$ of Data. arXiv:1203.3774 [hep-ex].

[9] M. Holthausen, K. S. Lim, and M. Lindner, Planck scale Boundary Conditions and the Higgs Mass. JHEP 1202 (2012) 037, arXiv:1112.2415 [hep-ph]

[10] J. A. M. Vermaseren, New features of FORM. arXiv:math-ph/0010025.

[11] S. G. Gorishnii, S. A. Larin, L. R. Surguladze, and F. V. Tkachov, MINCER: Program for multiloop calculations in quantum field theory for the SCHOONSCHIP system. Comput. Phys. Commun. 55 (1989) 381-408.

[12] M. Misiak and M. Munz, Two loop mixing of dimension five flavor changing operators. Phys. Lett. B344 (1995) 308-318, arXiv:hep-ph/9409454.

[13] K. G. Chetyrkin, M. Misiak, and M. Munz, Beta functions and anomalous dimensions up to three loops. Nucl. Phys. B518 (1998) 473-494, arXiv:hep-ph/9711266 [hep-ph].

[14] M. Steinhauser, MATAD: A program package for the computation of massive tadpoles. Comput. Phys. Commun. 134 (2001) 335-364, arXiv:hep-ph/0009029.

[15] P. Nogueira, Automatic Feynman graph generation.

J. Comput. Phys. 105 (1993) 279-289.

[16] T. Van Ritbergen, A. Schellekens, and J. Vermaseren, Group theory factors for Feynman diagrams. International Journal of Modern Physics A 14 (1999) no. 1, 41-96. cited By (since 1996) 70.

[17] G. 't Hooft and M. J. G. Veltman, Regularization and Renormalization of Gauge Fields. Nucl. Phys. B44 (1972) 189-213.

[18] E. Brezin, J. C. Le Guillou, J. Zinn-Justin, and B. G. Nickel, Higher oder contributions to critical exponents. Phys. Lett. 44A (1973) 227-228.

[19] E. Brezin, J. C. Le Guillou, and J. Zinn-Justin, Addendum to wilson's theory of critical phenomena and callan-symanzik equations in 4-epsilon dimensions.

Phys. Rev. D9 (1974) 1121-1124.

[20] M.-x. Luo and Y. Xiao, Two loop renormalization group equations in the standard model. Phys. Rev. Lett. 90 (2003) 011601, arXiv:hep-ph/0207271 [hep-ph].

[21] M. E. Machacek and M. T. Vaughn, Two-loop renormalization group equations in a general quantum field theory: (III). Scalar quartic couplings.

Nucl. Phys. B 249 (1985) no. 1, 70-92.

[22] M. E. Machacek and M. T. Vaughn, Two-loop renormalization group equations in a general quantum field theory (II). Yukawa couplings.

Nucl. Phys. B 236 (1984) no. 1, 221-232.

[23] M. Steinhauser, Higgs decay into gluons up to $O\left(\right.$ alpha**3(s) $\left.G(F) m^{* * 2}(t)\right)$. Phys.Rev. D59 (1999) 054005, arXiv:hep-ph/9809507 [hep-ph].

[24] O. Tarasov, A. Vladimirov, and A. Zharkov, The gell-mann-low function of QCD in the three-loop approximation. Phys. Lett. B 93 (1980) no. 4, 429-432.

[25] S. Larin and J. Vermaseren, The Three loop QCD Beta function and anomalous dimensions. Phys. Lett. B303 (1993) 334-336, arXiv: hep-ph/9302208 [hep-ph]. 
[26] D. J. Gross and F. Wilczek, Ultraviolet Behavior of Non-Abelian Gauge Theories. Phys. Rev. Lett. 30 (1973) 1343-1346.

[27] H. D. Politzer, Reliable Perturbative Results for Strong Interactions? Phys. Rev. Lett. 30 (1973) 1346-1349.

[28] M. Fischler and J. Oliensis, Two-loop corrections to the beta function for the Higgs-Yukawa coupling constant. Phys. Lett. B 119 (1982) no. 4, 385-386.

[29] D. R. T. Jones, Two-loop $\beta$ function for a $G_{1} \times G_{2}$ gauge theory. Phys. Rev. D 25 (1982) 581-582.

[30] I. Jack and H. Osborn, General background field calculations with fermion fields. Nucl. Phys. B 249 (1985) no. 3, 472-506.

[31] M. E. Machacek and M. T. Vaughn, Two-loop renormalization group equations in a general quantum field theory: (I). Wave function renormalization. Nucl. Phys. B 222 (1983) no. 1, 83-103.

[32] L. N. Mihaila, J. Salomon, and M. Steinhauser, Gauge coupling beta functions in the standard model to three loops. Phys. Rev. Lett. 108 (2012) 151602.

[33] L. Maiani, G. Parisi, and R. Petronzio, Bounds on the Number and Masses of Quarks and Leptons. Nucl. Phys. B136 (1978) 115.

[34] N. Cabibbo, L. Maiani, G. Parisi, and R. Petronzio, Bounds on the Fermions and Higgs Boson Masses in Grand Unified Theories. Nucl. Phys. B158 (1979) 295-305.

[35] M. Lindner, Implications of Triviality for the Standard Model. Z. Phys. C31 (1986) 295.

[36] T. Hambye and K. Riesselmann, Matching conditions and Higgs mass upper bounds revisited. Phys.Rev. D55 (1997) 7255-7262, arXiv:hep-ph/9610272 [hep-ph].

[37] N. Krasnikov, Restriction of the Fermion Mass in Gauge Theories of Weak and Electromagnetic Interactions. Yad. Fiz. 28 (1978) 549-551.

[38] P. Q. Hung, Vacuum Instability and New Constraints on Fermion Masses. Phys. Rev. Lett. 42 (1979) 873.

[39] H. D. Politzer and S. Wolfram, Bounds on Particle Masses in the Weinberg-Salam Model. Phys. Lett. B82 (1979) 242-246.

[40] M. Sher, Electroweak Higgs Potentials and Vacuum Stability. Phys. Rept. 179 (1989) 273-418.

[41] C. Ford, D. Jones, P. Stephenson, and M. Einhorn, The Effective potential and the renormalization group. Nucl. Phys. B395 (1993) 17-34. arXiv:hep-lat/9210033 [hep-lat].

[42] J. Elias-Miro, J. R. Espinosa, G. F. Giudice, G. Isidori, A. Riotto, et al., Higgs mass implications on the stability of the electroweak vacuum. Phys. Lett. B709 (2012) 222-228, arXiv:1112.3022 [hep-ph].

[43] Z.-z. Xing, H. Zhang, and S. Zhou, Impacts of the Higgs mass on vacuum stability, running fermion masses and two-body Higgs decays. arXiv:1112.3112 [hep-ph].

[44] J. Espinosa, G. Giudice, and A. Riotto, Cosmological implications of the Higgs mass measurement. JCAP 0805 (2008) 002, arXiv:0710.2484 [hep-ph].

[45] R. Hempfling and B. A. Kniehl, On the relation between the fermion pole mass and MS 
Yukawa coupling in the standard model. Phys.Rev. D51 (1995) 1386-1394, arXiv:hep-ph/9408313 [hep-ph].

[46] A. Sirlin and R. Zucchini, Dependence of the Higgs coupling hMS(M) on $m H$ and the possible onset of new physics. Nucl. Phys. B 266 (1986) no. 2, 389-409.

[47] K. Nakamura et al., The review of particle physics. J. Phys. G (2010) no. 37, 075021.

[48] M. Tentyukov and J. A. M. Vermaseren, The multithreaded version of FORM. arXiv: hep-ph/0702279.

[49] J. A. M. Vermaseren, Axodraw. Comput. Phys. Commun. 83 (1994) 45-58.

[50] F. Bezrukov, M. Y. Kalmykov, B. A. Kniehl, and M. Shaposhnikov, Higgs boson mass and new physics. arXiv:1205.2893 [hep-ph].

[51] G. Degrassi, S. Di Vita, J. Elias-Miro, J. R. Espinosa, G. F. Giudice, et al., Higgs mass and vacuum stability in the Standard Model at NNLO. JHEP 1208 (2012) 098, arXiv:1205.6497 [hep-ph].

[52] S. Alekhin, A. Djouadi, and S. Moch, The top quark and Higgs boson masses and the stability of the electroweak vacuum. Phys.Lett. B716 (2012) 214-219, arXiv:1207.0980 [hep-ph].

[53] I. Masina, The Higgs boson and Top quark masses as tests of Electroweak Vacuum Stability. arXiv:1209.0393 [hep-ph]. 